\title{
Penetration of a radio frequency electromagnetic field into a magnetized plasma: one-dimensional PIC simulation studies
}

\author{
Fumiko Otsuka ${ }^{1 *}$, Tohru Hada ${ }^{1}$, Shunjiro Shinohara ${ }^{2}$ and Takao Tanikawa ${ }^{3}$
}

\begin{abstract}
Penetration of a radio frequency ( $\mathrm{rf}$ ) electromagnetic field into a magnetized plasma is discussed by performing one-dimensional particle-in-cell (PIC) simulations. We consider two models: an electrostatic (ES) model using an external rf voltage and an electromagnetic (EM) model using an external rf current. The background magnetic field is perpendicular to the one-dimensional system. In the ES model, the external rf voltage across the plasma produces the rf electric field into the plasma. When $\omega_{r f}=\Omega_{e} / 2$, where $\omega_{r f}$ and $\Omega_{e}$ are the externally applied field and electron gyrofrequencies, respectively, the ion-rich sheath is formed due to the electron wall loss caused by the electron polarization drift. When $\omega_{r f}=\omega_{L H} / 2$, where $\omega_{L H}$ is the lower hybrid frequency, the electron-rich sheath is formed due to the ion wall loss caused by its larger gyroradius than the electron. In the EM model, an induced electromagnetic field via the external if current forms a standing wave in the plasma region bounded by the external antennas. When the diameter of the plasma is equal to the wave length, the spatial/temporal profiles of the rf standing waves are well explained by a cold collisionless plasma linear theory except for the existence of the sheath. When the rf magnetic field is increased to $10 \%$ of the background magnetic field, the waveforms become irregular and complex because of the boundary effects.
\end{abstract}

Keywords: Radio frequency electromagnetic field; Extrenal electrode; Magnetized plasma;

Electrodeless plasma thruster

\section{Background}

Wave penetration into a plasma is an important problem from the point of view of a fundamental physics and from numerous applications in various fields such as plasma production (Shinohara and Shamrai 2002; Shinohara et al. 2009) plasma heating, and confinement (Shoji 1980). In this paper, we consider the penetration of both electrostatic and electromagnetic waves into a magnetized plasma with a specific application in mind: the next-generation plasma thrusters. In many of the conventional electric (plasma) thrusters, such as the ion engine and Hall thruster, thrust is obtained by accelerating the

\footnotetext{
*Correspondence: otsuka@esst.kyushu-u.ac.jp

${ }^{1}$ Interdisciplinary Graduate School of Engineering Sciences, Kyushu University, Kasuga, Fukuoka, 816-8580, Japan

Full list of author information is available at the end of the article
}

plasma using a static electric field (Goebel and Katz 2008; Raitses and Fisch 2001). Such an electric field, however, is produced by electrodes situated inside the plasma, and thus, because of their contact with the plasma, electrode erosion cannot be avoided. To overcome this difficulty, we propose 'electrodeless' thrusters that have electrodes located outside the plasma so that there is no direct contact between the plasma and the electrodes (Helicon Electrodeless Advanced Thruster (HEAT) project (Shinohara et al. 2013)). In the electrodeless thrusters, either electrostatic or electromagnetic waves are emitted from antennas outside the plasma region, so they penetrate into the plasma and accelerate it. A very long thruster life span can be expected because it is not limited by the electrode erosion.

Three electrodeless plasma thrusters, in which the radio frequency (rf) electric field penetration is essential,

\section{Springer}

C 2015 Otsuka et al. This is an Open Access article distributed under the terms of the Creative Commons Attribution License (http://creativecommons.org/licenses/by/4.0), which permits unrestricted use, distribution, and reproduction in any medium, provided the original work is properly credited. 
are the Lissajous Helicon Plasma Accelerator (LHPA) (Matsuoka et al. 2012; Nakamura et al. 2012; Nishida et al. 2012), the Variable Specific Impulse Magnetoplasma Rocket (VASIMR) (Ando et al. 2005, 2006; Bering III et al. 2010), and the Ponderomotive Acceleration/Ion Cyclotron Resonance (PA/ICR) (Otsuka et al. 2013a, 2013b). First, the LHPA uses a Lorentz force which is a product of an azimuthal electron current and a radial magnetic field. In the LHPA, two sets of planar antennas are used to electrostatically excite a rotating electric field (REF), which produces the azimuthal electron current (Matsuoka et al. 2012). Second, the VASIMR uses the ion cyclotron resonance (ICR) for the ion acceleration (Bering III et al. 2010). In the VASIMR-type experiment, a helically wound antenna is used to electromagnetically excite an ion cyclotron wave propagating along the magnetic field line (Ando et al. 2005, 2006). Third, the ponderomotive acceleration (PA) uses a sign flipping of a ponderomotive potential near ion gyroresonance, leading to a unidirectional acceleration of ions (Dodin et al. 2004). We examined the PA combined with the ICR using the test particle simulations (Otsuka et al. 2013a, 2013b). In our test particle simulations, the rf electromagnetic fields are assumed to be present inside the plasma. This may well be an optimistic simplification because the plasma in general acts to shield the external waves from penetrating inside. Further, in the various experiments on the thrusters, it is difficult to observe the efficiency of the wave penetration, although it is directly related to the thruster's performance. In this paper, for applications to various types of electrodeless plasma thrusters in general, we investigate the rf electric field penetration by performing one-dimensional particlein-cell (PIC) simulations.

We study two models with different configurations of the external electrodes. The first is the electrostatic (ES) model in which an $\mathrm{rf}$ voltage difference is applied to a pair of planar electrodes to generate an rf electric field between the electrodes. The second is the electromagnetic (EM) model in which an rf current is applied to an external azimuthal coil which surrounds the plasma to generate the rf magnetic field and induced rf electric field inside the coil.

Our ES model is essentially the same as the one by Matsuoka et al. (2012) who developed a theoretical model in which the rf electric fields in a plasma and sheaths were derived in a high-frequency range of $\omega_{L H} \ll \omega_{r f} \ll \Omega_{e}$. Here, $\omega_{r f}, \omega_{L H}$, and $\Omega_{e}$ are an externally applied field frequency, lower hybrid frequency, and electron gyrofrequency, respectively. In this paper, we choose high and low frequencies for $\omega_{r f}$. The high frequency we use is $\omega_{r f}=$ $\Omega_{e} / 2$ to compare our numerical results with the theoretical model by Matsuoka et al. (2012). The low frequency we use is $\omega_{r f}=\omega_{L H} / 2$ to apply the PA/ICR scheme. For the PA scheme to operate, $\omega_{r f}$ should be near the ion gyrofrequency, $\Omega_{i}$, but we set $\omega_{r f}=\omega_{L H} / 2>\Omega_{i}$ as the low-frequency case, to reduce the computation time.

An ion-rich sheath, i.e., a positively charged boundary, and an electron-rich sheath, i.e., a negatively charged boundary, were studied using computer simulations and laboratory experiments. For example, the ion-rich sheaths were simulated around antennas immersed in space plasma (Miyake and Usui 2009; Usui et al. 2004). A transition from the ion-rich to the electron-rich sheath was experimentally observed, when a rapid rising voltage was applied to a plane gridded electrode in an unmagnetized discharge plasma (Stenzel et al. 2011). In our ES model, we show numerically that the electron-rich and ion-rich sheaths can be formed according to the value of $\omega_{r f}$.

Our EM model is similar to the one proposed by Ikeda et al. (2005) who focused on the evanescent rf wave with $\omega_{r f}>\omega_{L H}$; thus, the rf waves were highly damped near the plasma edge with a skin-depth scale and the spatial profile of the rf waves was independent of the plasma radius. Conversely, we focus on a propagating $\mathrm{rf}$ wave with $\omega_{r f}<\omega_{L H}$; thus, the rf wave excited on one side of the plasma boundary propagates toward the other side of the boundary as extraordinary waves. Accordingly, a standing wave is formed in the plasma region. In this paper, we compare spatial/temporal structures of the standing rf waves obtained from simulation and cold collisionless plasma linear theory (Hada et al. 2009; Stix 1992). Finally, we discuss which model should be adopted for application to the electrodeless plasma thrusters.

\section{Methods}

Figure 1 shows the ES (left) and EM (right) models we consider. Although the thruster in reality is cylindrical and three-dimensional as shown in the top of Fig. 1, we consider a simple, one-dimensional model, as shown at the bottom. The main axis is $x$, which corresponds to the radial direction in the realistic, three-dimensional cylindrical system. For both the ES and EM models, the external electrodes (shown by the yellow regions) are placed at the plasma boundaries of $x= \pm a$, where the radius of the plasma is $a$, the center of the plasma at $x=0$, and the background magnetic field with its magnitude, $B_{0}$, along the $z$-axis. The outside of the plasma is a vacuum, and further away lies a pair of absorbing boundaries. Some portion of the electromagnetic waves in the plasma is converted into the light wave in the vacuum and eventually lost at the absorbing boundaries. We use perfectly matched layers (PML) for the wave absorption at the boundaries.

The plasma consists of either argon or helium ions and electrons. Neutral particles are absent, and the collisionless plasma is assumed. For numerical computation, at every time step, the ions and electrons are accelerated by the Lorentz force, the charge and current densities 


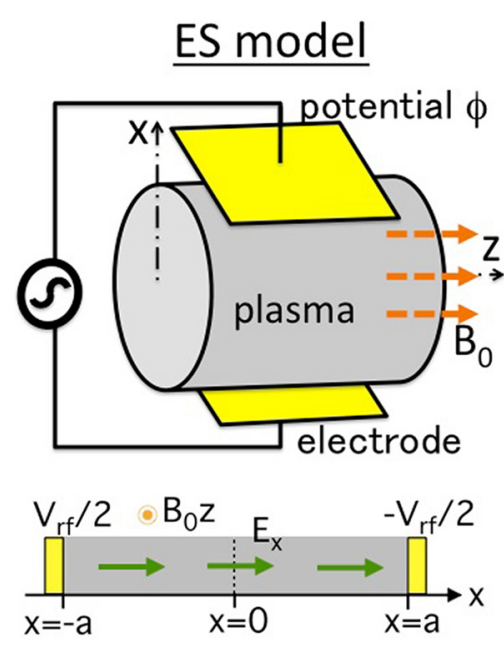

\section{EM model}
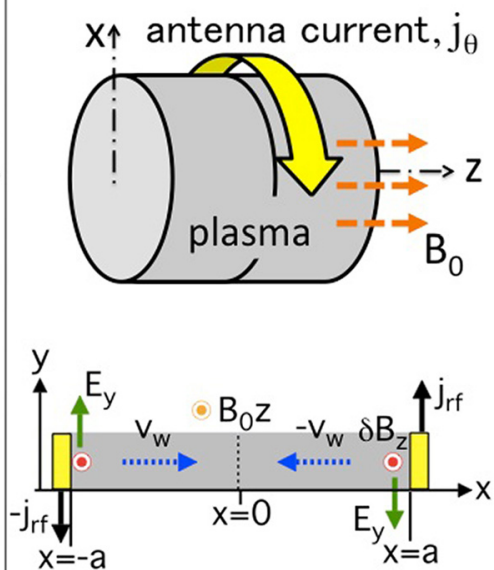

Fig. 1 Schematic pictures of simulation models. Cylindrical and three-dimensional plasma (in the top figures) are simplified as one-dimensional plasma (in the bottom figures). (Left) the electrostatic (ES) model using the external rf voltage difference, $V(t)=V_{r f} \cos \left(\omega_{r f} t\right)$, and (Right) the electromagnetic (EM) model using the external rf current density, $j_{y}( \pm a)= \pm j_{r f} \sin \left(\omega_{r f} t\right)$

evaluated, and the electromagnetic fields time-advanced using Maxwell's equations with a charge-conserving current deposition algorithm. The plasma is absorbed at the plasma boundaries of $x= \pm a$. We use VORPAL (Nieter and Cary, 2004) for the simulation code.

\section{Plasma parameters}

Plasma parameters in the ES and EM models are the same as follows. Initially, ions and electrons are uniformly distributed between $x=-a$ and $x=a$, and their velocities are given by the Maxwellian distribution with temperatures, $T_{i}=m_{i} v_{t h, i}^{2}$ and $T_{e}=m_{e} v_{t h, e}^{2}$, where $v_{t h, i}$ and $v_{t h, e}$ are ion and electron thermal velocities, respectively, and $m_{i}$ and $m_{e}$ the ion and electron mass, respectively. There are 200 macro-particles per cell for both ions and electrons.

In this paper, we investigate the wave penetration process, assuming that the same physical process dominates other parameters with different scales. Here, to save the computation time, we choose the parameters as follows: $m_{i} / m_{e}=200, T_{e}=T_{i}=0.0004 m_{e} c^{2}$, and $\Omega_{e} / \omega_{p e}=1$, where $c$ is the speed of light.

Under these parameters, the lower hybrid and ion gyrofrequencies normalized to the electron plasma frequency are $\omega_{L H} / \omega_{p e} \simeq 0.05, \Omega_{i} / \omega_{p e}=1 / 200$, respectively; then, $\omega_{L H} / \Omega_{i} \simeq 10$, where $\omega_{L H}^{-2}=\left(\Omega_{i} \Omega_{e}\right)^{-1}+$ $\left(\Omega_{i}^{2}+\omega_{p i}^{2}\right)^{-1}$. Alfvén and ion sound velocities normalized to the light speed are $v_{A} / c \simeq 0.07$ and $v_{S} / c \simeq 1.4 \times 10^{-3}$, respectively, where $v_{A}^{2} / c^{2} \simeq \Omega_{i}^{2} / \omega_{p i}^{2}$ and $v_{S}^{2} / c^{2}=T_{e} / m_{i} c^{2}$. Thus, $v_{A} \gg v_{S}$ represents the validity of the cold plasma approximation.
We perform a total of four runs whose parameters for the external fields are shown in Table 1. For all the runs, the spatial grid size and the time step size are the Debye length of $\Delta=v_{t h, e} / \omega_{p e}$ and $\Delta t=0.9 \Delta / c$ corresponding to $\omega_{p e} \Delta t=0.018$. For run 1 , the system length, $L$, (i.e., the plasma diameter, $2 a$ ) is $L=1000 \Delta$, which corresponds to $a=10 c / \omega_{p e}$, whereas for runs 2 to $4, L=767 \Delta$, which corresponds to $a \simeq 27 \rho_{i}$, where $\rho_{i}$ is the typical ion gyroradius defined by $\rho_{i}=v_{t h, i} / \Omega_{i}=\sqrt{m_{i} / m_{e}} \Delta$.

\section{Electrostatic (ES) model}

In the ES model, two planar electrodes at $x= \pm a$ are given time-varying electrostatic potentials, $\phi(-a)=$ $V_{r f} \cos \left(\omega_{r f} t\right) / 2$ and $\phi(a)=-V_{r f} \cos \left(\omega_{r f} t\right) / 2$. Here, an external voltage difference between the two electrodes is given by $V(t)=\phi(-a)-\phi(a)=V_{r f} \cos \left(\omega_{r f} t\right)$. The rf electric field is then generated into the plasma. For the evolution of the electric field, the Poisson equation is solved at every time step based on the instantaneous charge density distribution by VORPAL (Nieter and Cary, 2004). Hence, in the ES model, the electric field is exactly

Table 1 Four runs are performed, where the system lengths, $L=2 a$, are $1000 \Delta$ and $767 \Delta$ for run 1 and runs $2-4$, respectively

\begin{tabular}{llll}
\hline & Method & $\omega_{\text {rf }}$ & External field amplitude \\
\hline Run 1 & ES & $10 \omega_{L H}$ & $V_{\text {rf }} / C B_{0} L=1$ \\
Run 2 & ES & $0.5 \omega_{L H}$ & $V_{\text {rf }} / C B_{0} L=0.008$ \\
Run 3 & EM & $0.5 \omega_{L H}$ & $\mu_{0} j_{\text {rf }} \Delta / B_{0}=0.02$ \\
Run 4 & EM & $0.5 \omega_{L H}$ & $\mu_{0} j_{r f} \Delta / B_{0}=0.2$ \\
\hline
\end{tabular}


parallel to the $x$ direction, and there are no magnetic field fluctuations.

In a high-frequency range of $\omega_{L H} \ll \omega_{r f} \ll \Omega_{e}$, the electric field penetration in the ES model was discussed theoretically by Matsuoka et al. (2012). Here, we briefly review their theoretical model, in which all electrons move as a rigid box, ions are immobile, and there are no electrons in the sheath. Solving Poisson's equations in a region bounded by harmonic rf potentials, the electric fields, $E_{p}$, $E_{1 s}$, and $E_{2 s}$, in the plasma and in the left- and right-side sheaths are obtained by:

$$
\begin{aligned}
E_{p} & =E_{1}\left(1-\frac{2 \eta s_{0}^{2}}{V_{r f}}\right) \cos \left(\omega_{r f} t\right), \\
E_{1 s} & =\eta\left(x+a-s_{1}\right)+E_{p}, \\
E_{2 s} & =\eta\left(x-a+s_{2}\right)+E_{p},
\end{aligned}
$$

where $E_{1}=V_{r f} / 2 a$ is the rf electric field amplitude in a vacuum, $\eta=e n_{0} / \epsilon_{0}, n_{0}$ the plasma number density, $e$ the elementary charge, and $\epsilon_{0}$ the permittivity in the vacuum. The left- and right-side sheath widths, $s_{1}$ and $s_{2}$, are written by:

$$
\begin{aligned}
& s_{1}(t)=s_{0}\left(1-\cos \omega_{r f} t\right), \\
& s_{2}(t)=s_{0}\left(1+\cos \omega_{r f} t\right),
\end{aligned}
$$

where the excursion length of the electron polarization drift is:

$$
s_{0}=\frac{L}{4} \frac{\Omega_{e}^{2}}{\omega_{p e}^{2}}\left(\sqrt{\epsilon^{2}+q}-\epsilon\right),
$$

where $\epsilon=1-\omega_{r f}^{2} / \Omega_{e}^{2}, q=8\left(\omega_{p e} / \Omega_{e}\right)^{2}\left(\delta_{e} / L\right)$, and $\delta_{e}=$ $e V_{r f} / m_{e} \Omega_{e}^{2} L=E_{1} / \Omega_{e} B_{0}$, which represents an excursion length of the electron polarization drift for perfect penetration. Equation (1a) show that the amplitude of $E_{p}$ decreases with the increase in $s_{0}$, because of the shielding by the ion-rich sheath. In addition, (2) describes the maximal sheath width of $2 s_{0}$. The potentials corresponding to the electric fields (1) are shown in the 'Appendix'. Under the simulation parameters for run $1, \epsilon=3 / 4, q=8 / 20$, $\delta_{e}=c / \omega_{p e}$; the sheath width is then $2 s_{0} \simeq 2.3 c / \omega_{p e}$, and the reduction rate in (1a) is $2 \eta s_{0}^{2} / V_{r f} \simeq 0.13$. In the 'Results' section, we compare the numerical results in run 1 with the theories (1) to (3).

Using the ES model, we perform run 1 and run 2 in Table 1. We set two different $\omega_{r f}$ to examine the $\omega_{r f}$ dependence. In run 1 for a high-frequency case, we set $\omega_{r f}=\Omega_{e} / 2 \simeq 10 \omega_{L H}$ and $V_{r f}=c B_{0} L$. In run 2 for a low-frequency case, we set $\omega_{r f}=\omega_{L H} / 2 \simeq 5 \Omega_{i}$ and $V_{r f} \simeq 0.008 c B_{0} L$. In run 1, the larger $V_{r f}$ than that in run 2 increases the sheath width, so that the sheath behavior is visible in detail, and can be compared with the theory. In run 2, the smaller $V_{r f}$ produces $E_{1}$ which is equal to the amplitude of $E_{x}$ in run 3 based on the linear theory (6b), so that the results of the ES and EM models can be compared.

\section{Electromagnetic (EM) model}

In the EM model, a pair of currents in opposite directions is located at the plasma boundaries. The time-varying current densities at the left and right boundaries are given by $j_{y}(-a)=-j_{r f} \sin \left(\omega_{r f} t\right)$ and $j_{y}(a)=j_{r f} \sin \left(\omega_{r f} t\right)$, respectively, where the width of the antenna is $\Delta$. The timevarying $j_{y}$ produces the $z$ component of the rf magnetic field, $\delta B_{z}$, from Ampere's law:

$$
\delta B_{z}=B_{1} \sin \left(\omega_{r f} t\right),
$$

where $B_{1}=\mu_{0} j_{r f} \Delta / 2$, and $\mu_{0}$ is the absolute permeability of a vacuum.

Here, we describe a standing wave based on the cold collisionless plasma linear theory. In the EM model, the timevarying magnetic field $\left(\delta B_{z}\right)$ propagates into the plasma as an extraordinary wave, if the plasma diameter, $2 a$, is much larger than the wave length, $2 \pi / k$, where $k=\omega_{r f} / \nu_{w}$ is the wave number in the $x$ direction and $v_{w}$ is the wave phase velocity. The fluctuation of $\delta B_{z}$ traveling into the plasma is then written as:

$$
\begin{aligned}
& \delta B_{z}=-B_{1} \sin \left[k(x+a)-\omega_{r f} t\right], \\
& \delta B_{z}=B_{1} \sin \left[k(x-a)+\omega_{r f} t\right],
\end{aligned}
$$

where (5a) at $x=-a$ and (5b) at $x=a$ match (4). A linear superposition of these two anti-traveling waves produces the standing wave:

$$
\begin{aligned}
\delta B_{z} & =-2 B_{1} \cos (k x) \sin \left(k a-\omega_{r f} t\right), \\
E_{x} & =2 B_{1} v_{w} \sin (k x) \sin \left(k a-\omega_{r f} t\right)(D / S), \\
E_{y} & =-2 B_{1} v_{w} \sin (k x) \cos \left(k a-\omega_{r f} t\right),
\end{aligned}
$$

where (6c) is obtained from the Maxwell-Faraday's equation using (6a), and (6b) is obtained from the polarization of the transverse electric fields for the extraordinary wave (Stix 1992),

$$
\frac{i E_{x}}{E_{y}}=-\frac{D}{S} .
$$

In the above $S=(R+L) / 2$ and $D=(R-L) / 2$ and $R$ and $L$ are defined by (Hada et al. 2009; Stix 1992),

$$
\begin{aligned}
& R=1-\frac{\omega_{p e}^{2}+\omega_{p i}^{2}}{\left(\omega+\Omega_{i}\right)\left(\omega-\Omega_{e}\right)} \\
& L=1-\frac{\omega_{p e}^{2}+\omega_{p i}^{2}}{\left(\omega-\Omega_{i}\right)\left(\omega+\Omega_{e}\right)},
\end{aligned}
$$

where $\omega_{p e}$ and $\omega_{p i}$ are the electron and the ion plasma frequencies, respectively.

The left panel of Fig. 2 shows the cold plasma dispersion relation for the extraordinary wave in the frequency range below $\omega_{L H}$ under the plasma parameter we use. The vertical and horizontal axes are the frequency and 


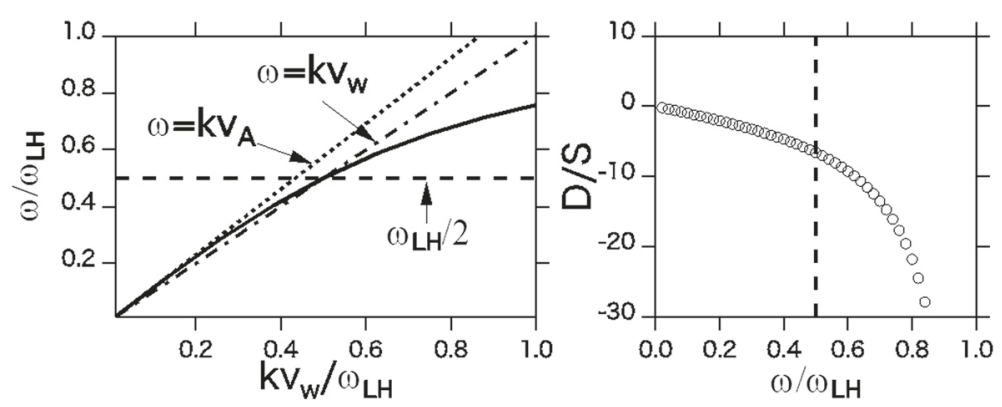

Fig. 2 Based on the cold plasma linear theory. (Left) cold plasma dispersion relation for the frequency below the lower hybrid frequency, $\omega_{L H}$, where the wave number $k$ is exactly perpendicular to the background magnetic field. (Right) polarization (7) of the transverse electric fields for the extraordinary wave as a function of the frequency

the wave number normalized to $\omega_{L H}$ and $\omega_{L H} / v_{w}$, respectively, where the phase velocity, $v_{w}$, satisfies the dispersion relation at $\omega=\omega_{L H} / 2$. The horizontal dashed line represents $\omega=\omega_{L H} / 2 \simeq 5 \Omega_{i}$. The dotted and chained lines represent $\omega=k v_{A}$ and $\omega=k v_{w}$, respectively. We obtain $v_{w} / c=0.06$ which is slightly less than $v_{A} / c=$ 0.07 because of the dispersion of the extraordinary wave. When $\omega \rightarrow 0, v_{w}$ approaches $v_{A}$.

The right panel of Fig. 2 shows the value of $D / S$ as a function of $\omega / \omega_{L H}$ by inserting the plasma parameter into (8). When $\omega<\omega_{L H}, D / S$ is negative so that $i E_{x} / E_{y}>0$. This means that the extraordinary wave for $\omega<\omega_{L H}$ is right-handed polarized. When $\omega=\omega_{L H} / 2$, we obtain $D / S \simeq-6.6$.

Using the EM model, we perform runs 3 and 4 in Table 1. We use two different external current density, $j_{r f}$, to examine the amplitude dependence of the rf wave, with $\omega_{r f}$ fixed at $\omega_{r f}=\omega_{L H} / 2$. Run 3 uses $\mu_{0} j_{r f} \Delta / B_{0}=0.02$ corresponding to $B_{1} / B_{0}=0.01$, whereas run 4 uses $\mu_{0} j_{r f} \Delta / B_{0}=0.2$ corresponding to $B_{1} / B_{0}=0.1$.

Note that the system length of $2 a=767 \Delta$ is equal to the wave length of $2 \pi / k$ where $k=\omega_{r f} / \nu_{w}, \omega_{r f}=$ $\omega_{L H} / 2, v_{w}=0.06 c$. Inserting $a k=\pi$ into (6), we then obtain the standing wave as:

$$
\begin{aligned}
\delta B_{z} & =-2 B_{1} \cos (\pi x / a) \sin \left(\omega_{r f} t\right), \\
E_{x} & =2 B_{1} v_{w} \sin (\pi x / a) \sin \left(\omega_{r f} t\right)(D / S), \\
E_{y} & =2 B_{1} v_{w} \sin (\pi x / a) \cos \left(\omega_{r f} t\right) .
\end{aligned}
$$

From the equations above, $E_{x}$ and $E_{y}$ have nodes at $x=$ $0, \pm a$, whereas $\delta B_{z}$ has anti-nodes at $x=0, \pm a$. In this paper, we compare the numerical results in runs 3 and 4 with the theory (9), where $v_{w} / c=0.06$ and $D / S=-6.6$ are used for the comparison. For run3 $\left(B_{1} / B_{0}=0.01\right)$, the amplitude of $E_{x}$ in (9b) is $2 B_{1} v_{w}|D / S| \simeq 0.008 c B_{0}$, which is equal to the vacuum amplitude of $E_{1}$ in run 2 , as described in the previous subsection.

\section{Results}

\section{ES model}

Figure 3 shows simulation results obtained from run 1 . In Fig. $3 \mathrm{a}$, the electric field, $E_{x}$, is plotted as a function of time, where the position of the measurement is $x=-3.5 c / \omega_{p e}$. The red solid and black dashed lines are obtained by the simulation and by the vacuum solution of $E_{x}=E_{1} \cos \left(\omega_{r f}\right) t$, respectively. The amplitude of $E_{x}$ is slightly less than $E_{1}$ as predicted by (1a). This result relates to the potential profile, as discussed later in this subsection.

Figure 3b-d show the spatial profiles of $E_{x}$; potential, $\phi$; and charge density, $\sigma$, normalized to $E_{1}, V_{r f}$, and $e n_{0}$, respectively. In these panels, the colored solid and dashed lines are obtained by the simulation and the theory from Matsuoka et al. (2012), respectively. In Fig. 3b-d, the measurement times are $\omega_{r f} t=4 \pi=12.6$ and $\omega_{r f} t=5 \pi=$ 15.7, which are displayed in Fig. 3a by the green and blue chained lines, respectively. At these times, $E_{x}$ in the vacuum are positively or negatively peaked in the time series of Fig. 3a. In Fig. 3b, the solutions (1) are depicted for two phases: $\left(s_{1}, s_{2}\right)=\left(0,2 s_{0}\right)$ for $V(t)=V_{r f}$ and $\left(s_{1}, s_{2}\right)=$ $\left(2 s_{0}, 0\right)$ for $V(t)=-V_{r f}$, indicated by the green and blue dashed lines, respectively. In Fig. 3c, the numerical potential profiles are obtained by the spatial integration of $E_{x}$ in Fig. 3b. The theoretical potential fields are plotted using (11). In addition to Fig. 3c, the vacuum solutions of $\phi(x)=$ $\mp\left(V_{r f} / 2\right)(x / a)$ are depicted by dotted lines. In Fig. 3d, the theoretical charge densities are $\sigma=e n_{0}$ in the sheath of the cathode side because all electrons move toward the anode side. The theoretical sheath regions with the scale of $2 s_{0} \simeq 2.3 c / \omega_{p e}$ are indicated by the gray shaded regions in Fig. 3b,c.

From the simulation result, we define the boundary between the plasma and sheath. When $|x|<5 c / \omega_{p e}, E_{x}$ approximates to a constant in Fig. 3b, and the charge neutrality is conserved, i.e., $\sigma$ is approximately 0 in Fig. $3 \mathrm{~d}$. Therefore, the plasma-sheath boundary is found to be $|x|=5 c / \omega_{p e}$, indicated by the vertical chained lines in 

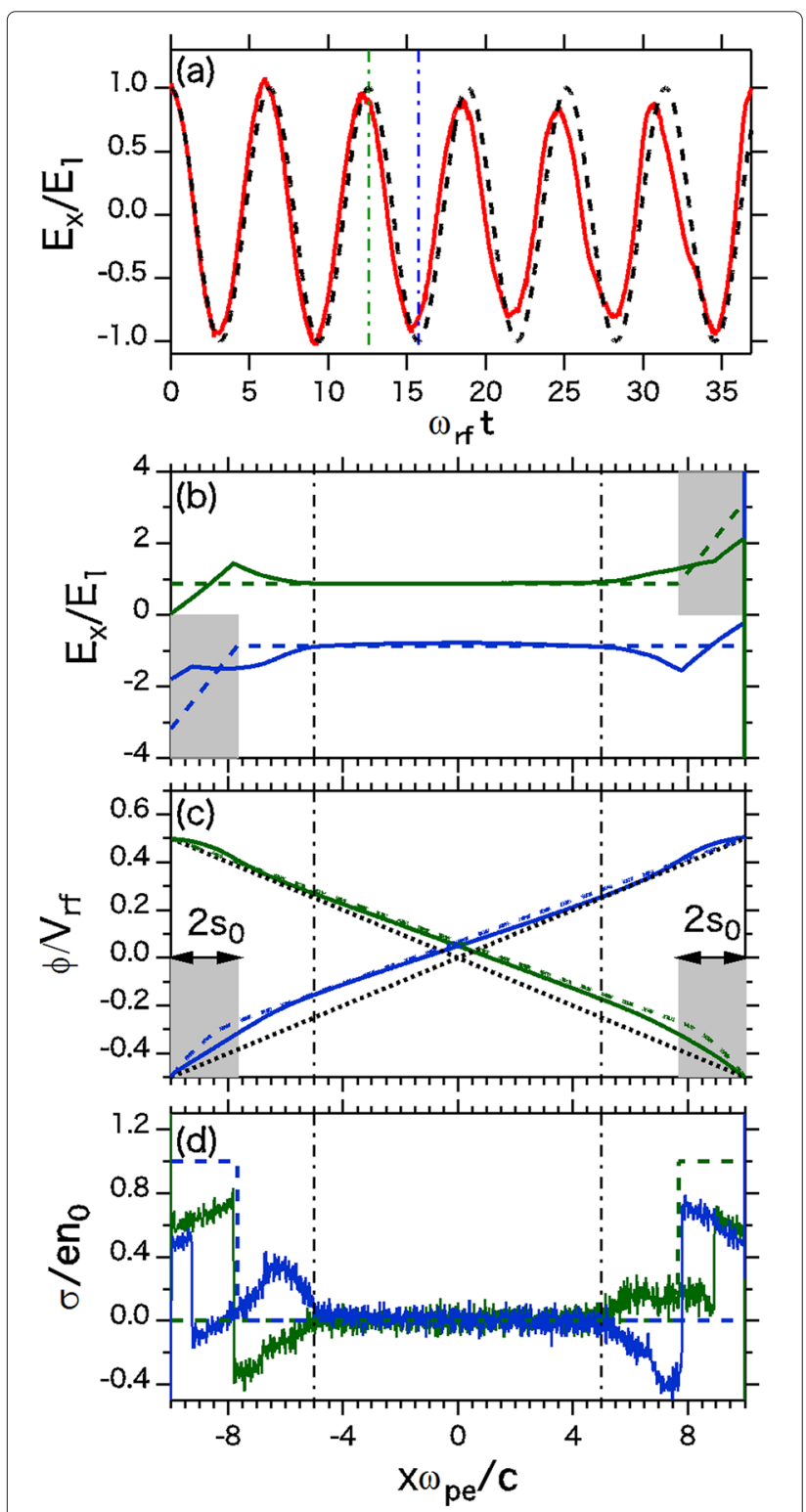

Fig. 3 Numerical results obtained from the ES model in the high-frequency case (run 1). a Electrostatic field $\left(E_{x}\right)$ as a function of time; spatial profiles of $\mathbf{b} E_{x} ; \mathbf{c}$ the electrostatic potential, $\phi$, obtained from the spatial integration of $E_{x}$; and $\mathbf{d}$ charge density, $\sigma$. The red solid line in $\mathbf{a}$ and the colored solid lines in $\mathbf{b}$ to $\mathbf{d}$ are obtained by the simulation. The black dashed line in $\mathbf{a}$ is the vacuum solution. The colored dashed lines in $\mathbf{b}$ to $\mathbf{d}$ are the solutions based on the theory of Matsuoka et al. (2012). The gray shaded region in $\mathbf{b}$ and $\mathbf{c}$ indicate the sheath region from the theory

Fig. 3b-d. Hence, the scale of the sheath width is found to be $5 c / \omega_{p e} \simeq 4.4 s_{0}$, which is 2.2 times larger than $2 s_{0} \simeq$ $2.3 c / \omega_{p e}$ obtained from the theory. In the plasma region of $|x|<5 c / \omega_{p e}$, the spatial profiles of $E_{x}$ and $\phi$ match with the theory. In the sheath region of $5 c / \omega_{p e}<|x|<$ $a=10 c / \omega_{p e}$, the value of $\sigma$ is locally positive or negative, and the value of $\phi$ is larger than the vacuum solution.
These results show that the ion-rich sheath is formed in the whole region of the sheath, although the negative charge density exists locally in the sheath. Therefore, the simulation result is qualitatively consistent with the theory by Matsuoka et al. (2012). In addition, we predict that the amplitude of the electric field (solid line) in Fig. 3a is less than $E_{1}$ (dashed line), because of the presence of the ion-rich sheath, which shield the applied field.

Figure 4 shows simulation results obtained from run 2 . Figure $4 \mathrm{a}$ is the same as Fig. $3 \mathrm{a}$ except that numerical $E_{x}$ is several times smoother than the original $E_{x}$ and the position of the measurement is $x \simeq-4.1 \rho_{i} \simeq-1.2 c / \omega_{p e}$. The time variation of $E_{x}$ penetrated into the plasma is similar to the solution in the vacuum. However, a slightly larger amplitude of $E_{x}$ than $E_{1}$ is observed at $\omega_{r f} t>15$. Figure $4 \mathrm{~b}-\mathrm{d}$ are the same as Fig. $3 \mathrm{~b}-\mathrm{d}$, except that the solution (1) and corresponding solutions of $\phi$ and $\sigma$ are not depicted in these panels because the solution (1) is only valid for $\omega_{L H} \ll \omega_{r f} \ll \Omega_{e}$. In Fig. 4b,c, the black dashed lines are the vacuum solutions. In Fig. $4 \mathrm{~d}$, the numerical $\sigma$ is several times smoother than the original $\sigma$. We find that the spatial profiles of $E_{x}$ and $\phi$ roughly match with the vacuum solutions, except near the sheath region. The potential, $\phi$, near the cathode side at $|x|=26 \rho_{i}$ is lower than the vacuum solution. Also, the charge densities are negative at $25 \rho_{i}<|x|<a=27 \rho_{i}$. These results suggest that the negatively charged boundary, i.e., electron-rich sheath, is generated due to the ion wall loss and that the sheath width is $2 \rho_{i}$ defined for the ion thermal velocity. In this sheath region, more ions than electrons are lost as a result of their larger gyroradii. Furthermore, the slightly increased amplitude over $E_{1}$ in Fig. $4 \mathrm{a}$ is explained by the spatial profile of $\phi$ with the electron-rich sheath. Namely, the electron-rich sheath tends to enlarge the applied field in the plasma, which is opposite to the case of the ion-rich sheath we discussed in the previous paragraph.

\section{EM model}

Figure 5 shows the electromagnetic field fluctuations: (left) $\delta B_{z}$, (middle) $E_{x}$, and (right) $E_{y}$, obtained from run 3 . In the top and bottom panels, the electromagnetic field fluctuations are plotted as functions of time and space, respectively. The magnetic and electric fields are normalized to $B_{1}$ and $v_{w} B_{1}$, respectively, where $B_{1}=0.01 B_{0}$ and $v_{w} \simeq 0.06 c$. In all the panels, the solid and dashed lines are obtained from the simulation and the cold plasma linear theory (9), respectively. We find that both of the time variations and the spatial profiles for the three components almost match the linear theory except for the existence of the sheath as seen in $E_{x}$ at the boundaries. In Fig. $5 \mathrm{a}-\mathrm{c}$, the positions of the measurements are $x=0$ and $x=-a / 2 \simeq$ $-13.5 \rho_{i}$ for $\delta B_{z}$ and $E_{x}$ and $E_{y}$ components, respectively. In Fig. $5 \mathrm{~d}-\mathrm{f}$, the times for the measurements are displayed 


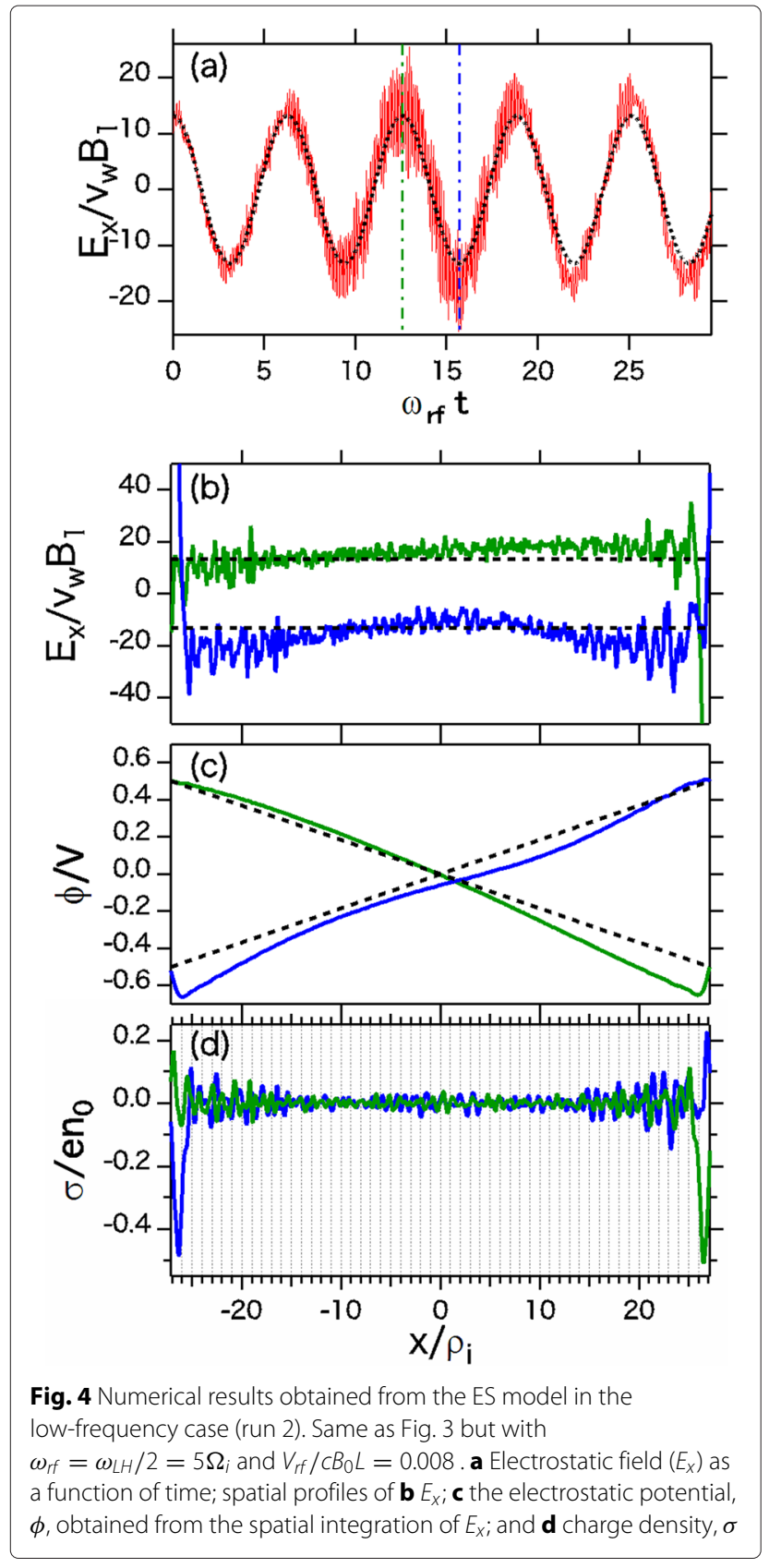

in the time series for each component in the top panels, by vertical chained lines with the same colors as the spatial profiles, for which the fluctuations are positively or negatively peaked in the time series.

Figure 6 is the same as the Fig. 5, but the external field amplitude is $B_{1}=0.1 B_{0}$ (run 4 ). We find that $\delta B_{z}, E_{x}$, and $E_{y}$ obtained from the simulation deviate from the linear theory. The waveforms are not simple sinusoidal but irregular and complex both in the spatial and temporal profiles. We also find that the sheath width in the $E_{x}$ component increases as $B_{1}$ increases.

\section{Discussion}

In this section, four issues from our analysis will be examined. First, we discuss the plasma wall loss process in the ES model. When $\omega_{r f}=\Omega_{e} / 2 \simeq 10 \omega_{L H}$ (the highfrequency case), we found the ion-rich sheath, which was qualitatively consistent with the theory from Matsuoka et al. (2012). As the theory predicted, mainly, electrons were lost because of the polarization drift, resulting in the ion-rich sheath. However, the sheath width obtained from the simulation was $4.4 s_{0}$, which was 2.2 times larger than from the theory, where $s_{0}$ is the excursion length of the electron polarization drift. In addition, a negative-charged density was locally observed in the sheath. These results were inconsistent with the theory. In run 1 , we used the external voltage as $V_{r f}=c B_{0} L$, which was larger than that used in the PIC simulation by Matsuoka et al. (2012). Therefore, the larger $V_{r f}$ may break the assumptions of immobile ions and rigid box motion of electrons. Hence, we suggest, for a strong external voltage, the mobile ions and electron motion in detail should be considered for a proper description of the sheath dynamics.

When $\omega_{r f}=\omega_{L H} / 2$ (the low-frequency case), we found the electron-rich sheath with the scale of $2 \rho_{i}$, where $\rho_{i}$ was ion thermal gyroradius. Here, we consider ion polarization drift as a candidate of the ion wall loss process in this case. When the rf electric field is perfectly penetrated in the plasma, the excursion length of the ion polarization drift can be written by $\delta_{i}=E_{1} / B_{0} \Omega_{i}$, where $E_{1}$ is the amplitude of the $\mathrm{rf}$ field in the vacuum. The excursion length normalized to the ion gyroradius, $\rho_{i}=v_{t h, i} / \Omega_{i}$, becomes:

$$
\frac{\delta_{i}}{\rho_{i}}=\frac{E_{1}}{B_{0} v_{t h, i}}=2 \frac{B_{1}}{B_{0}} \frac{v_{w}}{v_{t h, i}}\left|\frac{D}{S}\right| \sim 5.6 .
$$

Inserting the parameter of run 2 into $E_{1}$, we obtain $\delta_{i}=$ $5.6 \rho_{i}$. If ions are lost as a result of the ion polarization drift, the sheath width should have the scale of $2 \delta_{i} \sim 11 \rho_{i}$, which is larger than the sheath width of $2 \rho_{i}$ obtained from the simulation. Therefore, we conclude that the ion wall loss in the low-frequency case is not the result of the ion polarization drift but of the thermal ion gyromotion.

Second, we discuss the validity of the cold collisionless plasma linear theory in the EM model. We fixed the plasma radius at $a=\pi / k$ so that the rf electric fields were nodes at the boundaries within the linear theory. When the rf field amplitude was small, with $B_{1}=0.01 B_{0}$, the simulation result matched the theory except for the existence of the sheath, and the rf standing waves were stably excited in the plasma. When the rf amplitude was increased to $B_{1}=0.1 B_{0}$, the waveforms became complex, as well as time evolutions of the electromagnetic fields measured at fixed spatial locations (Fig. 6a-c). The complexity may be due to the superposition effect with a 

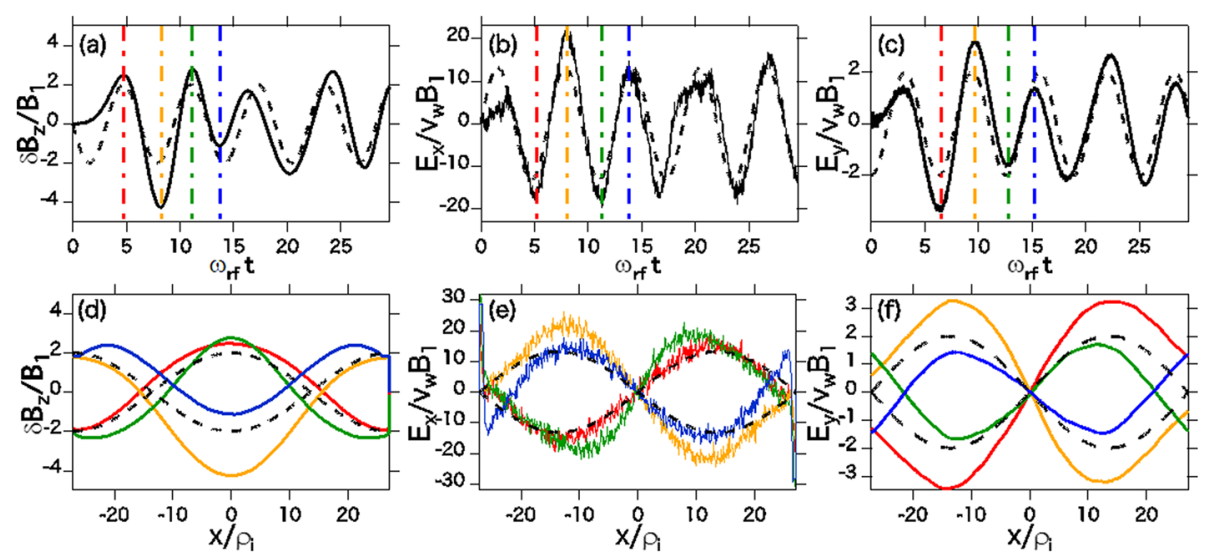

Fig. 5 Numerical results obtained from the EM model for $B_{1}=0.01 B_{0}$ (run 3). The black solid lines in $\mathbf{a}-\mathbf{c}$ show the time series of the if electromagnetic field fluctuations, $\delta B_{z}, E_{x}$ and $E_{y}$, excited into the plasma. The colored solid lines in $\mathbf{d}-\mathbf{f}$ show the spatial profiles of $\delta B_{z}, E_{x}$, and $E_{y}$, respectively, where the measurement times are displayed by the vertical chained lines with the same colors as the top panels. The black dashed lines in $\mathbf{a}-\mathbf{f}$ are the solutions (9) based on the cold collisionless plasma linear theory

reflected wave at the boundary or due to the plasma compression from incident waves as seen in the plot of $E_{x}$ in Fig. 6e. A study of the boundary effects including a correct sheath physics is necessary for a proper description of the electromagnetic wave penetration into a plasma. In addition, a collisional effect, especially electron-neutral collisions, cannot be ignored in the high-density plasma produced in laboratories. The collisional effect may prevent the stable excitation of the rf standing waves into the plasma. For future work, we need to investigate whether the rf standing wave can survive in the plasma, without a strong damping from the collisions.

Third, we apply our results to the plasma acceleration in the electrodeless plasma thruster. For efficient acceleration of the plasma, the rf electric field should penetrate deeply into the plasma center because the plasma density is maximum at the center and low near the edge for typical cylindrical plasma produced in laboratories. In the present PIC simulation, when $\omega_{r f}=\omega_{L H} / 2$, the rf electric fields penetrated into the plasma in both the ES and EM models. However, the spatial profiles were different. Namely, the ES model produced the nearly uniform $\mathrm{rf}$ electric field in the plasma, whereas the EM model produced the standing $\mathrm{rf}$ wave with a very small electric field at the center because of the symmetry. Therefore, we suggest that the ES model, which produces the rf field more deeply into the plasma, is more suitable for the external electrode configuration than the EM model. In addition, the electron-rich sheath in the low-frequency regime tends to cause an increase in the rf field amplitude in the plasma, whereas the ion-rich sheath in the high-frequency regime tends to cause a decrease. Therefore, the ES model
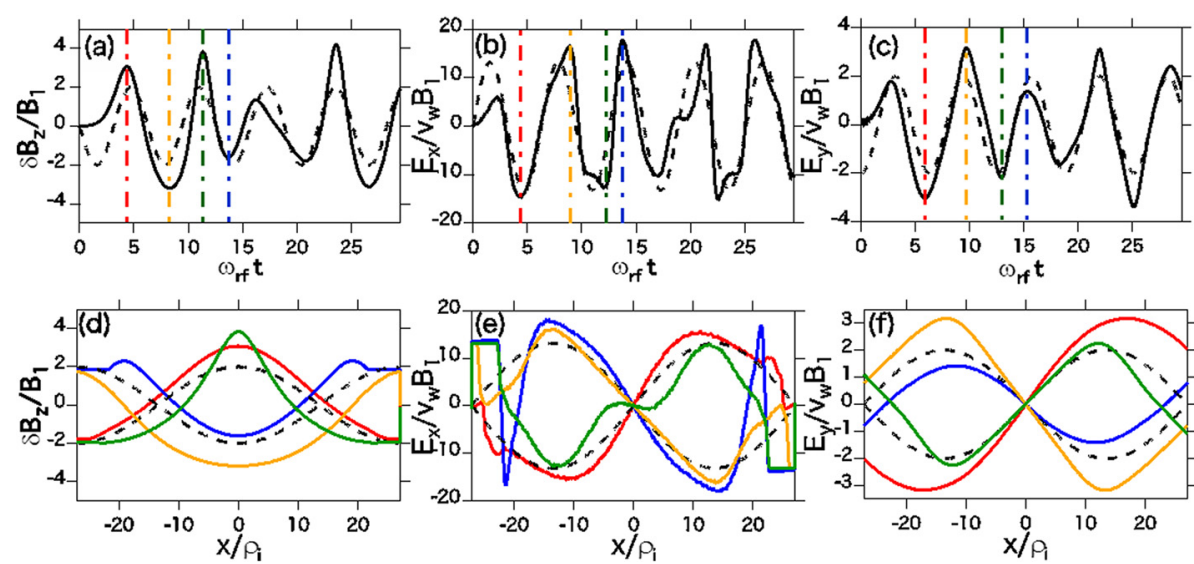

Fig. 6 Numerical results obtained from the EM model for $B_{1}=0.1 B_{0}$ (run 4). Same as Fig. 5 but with $B_{1}=0.1 B_{0}$. The black solid lines in $\mathbf{a}-\mathbf{c}$ show the time series of the rf electromagnetic field fluctuations, $\delta B_{z}, E_{x}$, and $E_{y}$, excited into the plasma. The colored solid lines in $\mathbf{d}-\mathbf{f}$ show the spatial profiles of $\delta B_{z}, E_{x}$, and $E_{y}$, respectively, where the measurement times are displayed by the vertical chained lines with the same colors as the top panels. The black dashed lines in a-f are the solutions (9) based on the cold collisionless plasma linear theory 
in the low-frequency regime can produce larger amplitude of the rf electric field than in the high-frequency regime, leading to efficient plasma acceleration.

Finally, we discuss the validity of our one-dimensional model, in which the plasma is only bounded in the $x$ axis. In the electrodeless plasma thrusters, the plasma is confined in a cylindrical boundary. This geometrical difference may lead to an essential difference for the rf field penetration process. For example, a one-dimensional fluid simulation for the electrostatic field penetration has indicated that the field penetration in the cylindrical geometry was reduced by about $6 \%$ in comparison with a planar model (priv. comm. with K. P. Shamrai). Therefore, to make more realistic estimates, the wave penetration into a cylindrical plasma should be investigated for the development of the electrodeless plasma thrusters.

\section{Conclusions}

We investigated the external radio frequency (rf) electromagnetic field penetration into the magnetized collisionless plasma using one-dimensional particle-in-cell (PIC) code, which simulates the plasma and electromagnetic field evolution in a direction perpendicular to the background magnetic field. We considered two different electrode configurations: an electrostatic (ES) model using an external rf voltage and an electromagnetic (EM) model using an external rf current.

In the ES model, the external rf voltage across the plasma generates the electrostatic field in the plasma. The sheath behavior depends on the externally applied rf field frequency, $\omega_{r f}$. When $\omega_{r f}=\Omega_{e} / 2$ (the high-frequency case), an ion-rich sheath appeared near both the anode and cathode sides of the electrodes with several scales of the electron polarization drift, because more electrons than ions are lost as a result of the electron polarization drift. The penetrated rf electric field was qualitatively consistent with the theoretical model (Matsuoka et al. 2012), but the details of the sheath did not match the model. When $\omega_{r f}=\omega_{L H} / 2$ (the low-frequency case), an electron-rich sheath appeared near the cathode side of the electrode with a scale similar to the thermal ion gyroradius, because more ions were lost due to their larger gyroradii than electrons.

In the EM model, the standing electromagnetic rf waves were formed as a result of superposition of two antitraveling extraordinary waves excited by the external $\mathrm{rf}$ current. When the plasma diameter was equal to the wave length, the rf electric field at the plasma boundaries were nodes in the cold collisionless plasma linear theory. For a small amplitude of the external rf field with $1 \%$ of the background magnetic field amplitude, the spatial/temporal profiles of the $\mathrm{rf}$ standing waves, except for the sheath, approximately matched the linear theory. When the amplitude of the external rf field increased to
$10 \%$ of the background field amplitude, the waveforms were irregular and complex due to the boundary effects.

\section{Appendix}

We describe the potential profile corresponding to (1) in the matrix sheath model developed by Matsuoka et al. (2012). Poisson's equations are solved including the ionrich sheath. The potentials, $\phi_{p}, \phi_{1 s}$, and $\phi_{2 s}$, in the plasma and in the left- and right-side sheath, are then obtained by:

$$
\begin{aligned}
\phi_{p} & =A_{p} x+B_{p},\left(-a+s_{1} \leq x \leq a-s_{2}\right), \\
\phi_{1 s} & =-\frac{\eta}{2} x^{2}+C_{1} x+D_{1},\left(-a \leq x<-a+s_{1}\right), \\
\phi_{2 s} & =-\frac{\eta}{2} x^{2}+C_{2} x+D_{2},\left(a-s_{2}<x \leq a\right),
\end{aligned}
$$

where $\eta=e n_{0} / \epsilon_{0}, a$ is the plasma radius, $s_{1}$ and $s_{2}$ the leftand right-side sheath widths defined by (2), and the other coefficients are as follows:

$$
\begin{aligned}
& A_{p}=-\frac{1}{L}\left[V(t)+\eta \frac{s_{1}^{2}-s_{2}^{2}}{2}\right], \\
& B_{p}=\frac{\eta}{4}\left(s_{1}^{2}+s_{2}^{2}\right), \\
& C_{1}=\left(-\frac{L}{2}+s_{1}\right) \eta-\frac{1}{L}\left[V(t)+\eta \frac{s_{1}^{2}-s_{2}^{2}}{2}\right], \\
& C_{2}=\left(\frac{L}{2}-s_{2}\right) \eta-\frac{1}{L}\left[V(t)+\eta \frac{s_{1}^{2}-s_{2}^{2}}{2}\right], \\
& D_{1}=-\frac{\eta L^{2}}{8}+\frac{\eta L s_{1}}{2}-\eta \frac{s_{1}^{2}-s_{2}^{2}}{4}, \\
& D_{2}=-\frac{\eta L^{2}}{8}+\frac{\eta L s_{2}}{2}+\eta \frac{s_{1}^{2}-s_{2}^{2}}{4},
\end{aligned}
$$

where $V(t)$ is the external $\mathrm{rf}$ voltage with $V(t)=$ $V_{r f} \cos \left(\omega_{r f} t\right)$ and $L(=2 a)$ system length.

\section{Competing interests}

The authors declare that they have no competing interests.

\section{Authors' contributions}

FO carried out the simulation, compared it with the theoretical model, and drafted the manuscript. TH provided direction to this work, especially for the simulation and theoretical model. SS and TT provided direction to this work, especially from the experimental view point. All authors discussed the results and implications in this study. All authors read and approved the final manuscript.

\section{Acknowledgements}

This work was partly supported by the Grants-in-Aid for Scientific Research under Contract No. (S) 21226019 from the Japan Society for the Promotion of Science.

\section{Author details}

${ }^{1}$ Interdisciplinary Graduate School of Engineering Sciences, Kyushu University, Kasuga, Fukuoka, 816-8580, Japan. ${ }^{2}$ Institute of Engineering, Tokyo University of Agriculture and Technology, Koganei, Tokyo 184-8588, Japan. ${ }^{3}$ Research Institute of Science and Technology, Tokai University, Hiratsuka, Kanagawa 259-1292, Japan. 
Received: 3 October 2014 Accepted: 7 May 2015

Published online: 06 June 2015

\section{References}

Ando A, Hattori K, Inutake M (2005) Present status and future prospect of the VASIMR type plasma thruster. In: Proceedings of Space Plasma Symposium, 2005, JAXA-SP-05-020, in Japanese (JAXA). pp 64-69

Ando A, Hosokawa Y, Hatanaka M, Tobari H, Hattori K, Inutake M (2006) Ion heating experiment by a helical antenna in a fast-flowing plasma. Thin Solid Films 506-507:601-604

Bering IIIEA, Chang Díaz FR, Squire JP, Glover TW, Carter MD, McCaskill GE, Longmier BW, Brukardt MS, Chancery WJ, Jacobson VT (2010) Observations of single-pass ion cyclotron heating in a trans-sonic flowing plasma. Phys Plasmas 17:043509-1-19

Dodin IY, Fisch NJ, Rax JM (2004) Ponderomotive barrier as a Maxwell demon. Phys Plasmas 11:5046-5064

Goebel DM, Katz I (2008) Fundamentals of electric propulsion: ion and hall thrusters, book section chapter 1,4 and 7. Wiley Jet Propulsion Laboratory California Institute of Technology

Hada T, Shinohara S, Toki K, Tanikawa T, Funaki I (2009) Theoretical modelings of next generation electrodeless plasma thrusters driven by external electromagnetic field. In: Proceedings of Space Plasma Symposium FY2007 and FY2008, JAXA-RR-09-003, in Japanese (JAXA)

Ikeda Y, Hada T, Matsukiyo S, Shinohara S (2005) Response of a cylindrical plasma to time-varying external electromagnetic field: numerical simulation studies. In: Proceedings of 29nd Int. Electric Propul. Conf., IEPC-2005, Princeton University, October 31, 2005, IEPC-2005-177

Matsuoka T, Rudenko TS, Funaki I, Shamrai KP, Nakamura T, Nishida H, Tanikawa T, Hada T, Shinohara S (2012) One dimensional modeling of radio frequency electric field penetration into magnetized plasmas. Jpn J Appl Phys:096201

Miyake Y, Usui H (2009) New electromagnetic particle simulation code for the analysis of spacecraft-plasma interactions. Phys Plasmas:062904

Nakamura T, Yokoi K, Nishida H, Matsuoka T, Funaki I, Shinohara S, Tanikawa T, Hada T, Motomura T, Shamrai KP, Rudenko TS (2012) Study on helicon plasma Lissajous acceleration for electrodeless electric propulsion. Trans JSASS Aerospace Tech Japan 10:Tb-17

Nishida H, Nakamura T, Shinohara S, Matsuoka T, Funaki I, Tanikawa T, Hada T, Shamrai KP (2012) Study on proof-of-principle of Lissajous acceleration for electrodeless helicon plasma thruster. Front Appl Plasma Technol 5:67-72

Otsuka F, Hada T, Shinohara S, Tanikawa T (2013a) Numerical studies of ponderomotive acceleration and ion cyclotron resonance: application to next generation electric thrusters. Plasma Fusion Res 1406012:8

Otsuka, F, Hada T, Shinohara S, Tanikawa T (2013b) Numerical modeling of electrodeless electric thruster by ion cyclotron resonance/ponderomotive acceleration. Plasma Fusion Res 2406067:8

Raitses Y, Fisch NJ (2001) Parametric investigations of a nonconventional hall thruster. Phys Plasmas 8:2579

Shinohara S, Shamrai KP (2002) Effect of electrostatic waves on a rf field penetration into highly collisional helicon plasmas. Thin Solid Films 407:215-220

Shinohara S, Hada T, Motomura T, Tanaka K, Tanikawa T, Toki K, Tanaka Y, Shamrai KP (2009) Development of high-density helicon plasma sources and their applications. Phys Plasmas 057104:16

Shinohara S, Nishida H, Yokoi K, Nakamura T, Tanikawa T, Hada T, Otsuka F, Motomura T, Ohno E, Funaki I, Matsuoka T, Shamrai KP, Rudenko TS (2013) High-density helicon plasma sources: basics and application to electrodeless electric propulsion. Trans Fusion Sci Technol 63:164

Shoji T (1980) Description of radio-frequency plugging and heating in terms of plasma impedance. J Phys Soc Jpn 49:327-333

Stenzel RL, Gruenwald J, Ionita C, Schrittwieser R (2011) Electron-rich sheath dynamics. I. Transient currents and sheath-plasma instabilities. Phys Plasmas 062112:18

Stix TH (1992) Waves in plasmas. Springer-Verlag New York, New York

Usui H, Matsumoto H, Yamashita F, Yamamoto A, Omura Y (2004) Antenna analysis in magnetized plasma via particle-in-cell simulation. Adv Space Res 34:2433-2436

Nieter C, Cary JR (2004) VORPAL: a versatile plasma simulation code. J Comp Phys 196:448-473

\section{Submit your manuscript to a SpringerOpen ${ }^{\circ}$ journal and benefit from:}

- Convenient online submission

- Rigorous peer review

- Immediate publication on acceptance

- Open access: articles freely available online

- High visibility within the field

- Retaining the copyright to your article

Submit your next manuscript at $>$ springeropen.com 